\title{
Characteristic dynamic differences between healthy and low back pain subjects
}

\author{
M Nissan*,1, K Bar-Ilan ${ }^{1}, \mathrm{~S}$ Brown $^{2}$, E Luger $^{1}$, E Steinberg $^{1}$ and S Dekel ${ }^{1}$ \\ ${ }^{1}$ The Orthopaedic B' Department, Tel-Aviv Sourasky Medical Center, Sackler Faculty of Medicine, Tel-Aviv \\ University, Tel-Aviv, Israel; ${ }^{2}$ Physical Therapy Institute, Tel-Aviv Sourasky Medical Center, Sackler Faculty of \\ Medicine, Tel-Aviv University, Tel-Aviv, Israel
}

\begin{abstract}
Study design: Open retrospective study.
Objectives: To assess the functional capacity impairment of chronic low back pain (LBP) patients using characteristic dynamic changes.

Setting: Orthopaedics and Physiotherapy departments at Tel-Aviv, Israel.

Methods: Thirty-eight normal healthy volunteers and 607 chronic LBP patients were tested on a computerized 3-D lumbar dynamometer. The four major parameters measured were the maximal isometric torque (MIT), maximal velocity against $25 \%$ MIT and 50\% MIT $\left(\mathrm{MV}_{25}\right.$, $\mathrm{MV}_{50}$, respectively) and maximal torques in secondary axes (MST). All patients parameters were compared to the normal, healthy findings.

Results: All four parameters were found to be significantly different between healthy (or nonsymptomatic) and symptomatic LBP subjects.

Conclusions: The findings support the use of 3-D dynamometry and the four parameters mentioned to objectively classify patients with functional disability.

Spinal Cord (2000) 38, 414-419
\end{abstract}

Keywords: LBP; isometric torque; 3-D dynamometry; functional capacity impairment

\section{Introduction}

Low back pain (LBP) is a major, widespread medical problem in the modern western world. Up to $80 \%$ of the population are expected to have at least one episode of back pain in their lifetime. ${ }^{1}$ The medical profession as a whole treats more patients suffering from LBP than almost any other symptom or disease. The tremendous economic impact of $\mathrm{LBP}^{2,3}$ has led to many research projects and reports on this subject. The ongoing need for objective testing of functional disability related to chronic LBP is encouraging the search for new objective assessment techniques. One method which has been proposed for this purpose is 3D lumbar dynamometry.

The functional disability caused by LBP has a major influence on many aspects of the patient's quality of life, hence the importance of identifying the etiology and the severity of the condition. ${ }^{4}$ Frequently, the etiology of LBP remains unknown, the exceptions being cases with spinal cord and/or surrounding tissue damage that are anatomically related to the clinical symptoms. Some of the activities known to be associated with LBP include intensive activity invol-

*Correspondence: M Nissan, Orthopaedics B', Tel-Aviv Sourasky Medical Center, 6 Weizman Street, Tel Aviv 64329, Israel ving the back, the lifting of heavy loads, poor sitting posture and accidental back injury. ${ }^{1}$

The use of imaging methods such as X-ray, CT and MRI can help in the diagnosis of the anatomical abnormalities, but they contribute little to the understanding of the actual functional disability. ${ }^{5,6}$ Systems such as computerized dynamometers and electromyography (EMG) can add valuable objective, dynamic data that can help the clinician to define and assess the low back condition ${ }^{7-10}$ but they reveal little of the actual disabilities.

Currently, low back functional disability is assessed quantitatively by a number of techniques. These include isometric, isokinetic, isoinertial (keeping a constant resisting torque) and free movement methods. ${ }^{3,8,10,11}$ Common parameters measured by most of these systems are the maximal isometric muscle forces or torques (MIF or MIT) in one to three planes of motion. The range of motion (RoM) is often mentioned as well, ${ }^{12}$ but it is highly dependent on the measuring system, the patient's cooperation, body positioning and restraining, the patient's anatomy, and other variables. As a result, RoM is considered by many researchers as a non-comparable parameter in vivo. ${ }^{13-15}$ Other parameters, such as speed or MIF (measured in some non-standard positions) are measured very accurately and repeatedly by some 
systems but, while they might be recommended for the assessment of low back performance, ${ }^{12,14}$ they are specific to the system and protocol in use.

In the present study, we used a system that enables simultaneous three-dimensional (3-D) measurements of RoM, MIT, speeds and dynamic torque in a highly accurate and repeatable manner ${ }^{15-17}$ that enables inter-patient comparisons. Our findings support the use of the system to assist in LBP patient evaluation.

In the present study we were looking at the patients' disabilities as presented in the clinic and tests. We did not try to assess cooperation and effort or detect exaggeration. The same system can be used to assess the effort and cooperation of the tested subjects ${ }^{8}$ and our experience in these areas will be published in the future.

\section{Materials and methods}

\section{Study population}

We evaluated 607 patients suffering from chronic LBP who were referred to our clinic for a complementary mechanical assessment of functional disability, most of them being involved in social security or compensation claims. They came from all the strata of society, and their vital statistics are described in Table 1. The patients suffer from continuous or intermittent disabling LBP for at least 6 months and up to 40 years prior to testing. All patients had imaging procedures, electromyography (EMG) and relevant clinical tests in the past and had been treated conservatively and/or surgically for a wide variety of symptoms and etiologies (or suspected etiologies) as described in Table 2. The control group was made of 38 volunteers who were clinically healthy and had no back complaints during the 6 months prior to testing.

\section{The 3-D computerized dynamometer}

The IsoStation B-200 (Isotechnologies Co., NC, USA) used in this study is a multiple-axis isoinertial dynamometric device designed for measuring 3-D movements of the lower back. The B-200 simultaneously measures RoM, torques and angular velocities in the sagittal, coronal and transverse planes. Maximal isometric torques (MIT) are measured first. The angular velocities $\mathrm{MV}_{25}$ and $\mathrm{MV}_{50}$ developed in the major planes of movement against $25 \%$ and $50 \%$ of MIT are measured next. The secondary muscles torques MST (applied by agonist muscles and some projections of the primary muscles in secondary planes while moving and measuring $\mathrm{MV}_{25}$ and $\mathrm{MV}_{50}$ in the primary plane) are measured simultaneously in both secondary planes.

All the 645 study subjects had no contraindications for undergoing the test. These contraindications are mainly mechanical spinal instability or acute phase of a back pathology. The following is the standard test

Table 1 The study and the OOC populations

\begin{tabular}{|c|c|c|c|c|c|c|}
\hline & & $\mathrm{n}$ & Pat & Age (years) & $H(\mathrm{~cm})$ & $W(\mathrm{~kg})$ \\
\hline \multirow[t]{4}{*}{ Healthy subjects } & $\mathrm{F}-$ our & 11 & 0 & $29 \pm 7$ & $166 \pm 5$ & $57 \pm 4$ \\
\hline & $\mathrm{F}-\mathrm{OOC}^{*}$ & 62 & 0 & $3 \overline{2}$ & $16 \overline{4}$ & $6 \overline{5}$ \\
\hline & $\mathrm{M}-$ our & 27 & 0 & $34 \pm 12$ & $179 \pm 6$ & $81 \pm 14$ \\
\hline & $\mathrm{M}-\mathrm{OOC}^{*}$ & 62 & 0 & 36 & 175 & 81 \\
\hline \multirow[t]{4}{*}{ LBP patients } & $\mathrm{F}-$ our & 75 & $9 \pm 7$ & $36 \pm 14$ & $162 \pm 13$ & $62 \pm 12$ \\
\hline & $\mathrm{F}-\mathrm{OOC}^{*}$ & 49 & & 37 & 163 & 71 \\
\hline & $\mathrm{M}-$ our & 532 & $12 \pm 7$ & $37 \pm 11$ & $176 \pm 13$ & $80 \pm 13$ \\
\hline & $\mathrm{M}-\mathrm{OOC}^{*}$ & 55 & & $\overline{37}$ & $17 \overline{7}$ & $\overline{86}$ \\
\hline
\end{tabular}

$\mathrm{LBP}=$ lower back pain; $\mathrm{Pat}=$ pathologic parameters; $\mathrm{H}=$ height; $\mathrm{W}=$ weight; $\mathrm{F}=$ female; $\mathrm{M}=$ male. $*$ The $\mathrm{OCC}$ data does not include SD nor the number of Pat in the LBP patients

Table 2 The main diagnoses in the study population

\begin{tabular}{|c|c|c|c|c|c|c|c|c|c|}
\hline & Pat & $\underset{\mathrm{n}}{D D W H}$ & $\begin{array}{c}\text { Func } \\
\mathrm{n}\end{array}$ & $\begin{array}{c}H I D \\
\mathrm{n}\end{array}$ & $\begin{array}{c}L S \\
\mathrm{n}\end{array}$ & $\begin{array}{c}P L S \\
\mathrm{n}\end{array}$ & $\begin{array}{c}\text { Others* } \\
\mathrm{n}\end{array}$ & $\begin{array}{c}N S \\
\mathrm{n}\end{array}$ & Sum \\
\hline \multirow[t]{2}{*}{ Male } & 0 & 1 & 2 & 23 & 3 & 1 & 9 & 23 & 62 \\
\hline & $>0$ & 40 & 72 & 232 & 37 & 45 & 67 & 4 & 497 \\
\hline \multirow[t]{2}{*}{ Female } & 0 & - & 1 & 4 & 3 & 1 & 1 & 11 & 21 \\
\hline & $>0$ & 2 & 13 & 26 & 4 & 8 & 12 & - & 65 \\
\hline Total & & 43 & 88 & 285 & 47 & 55 & 89 & 38 & 645 \\
\hline
\end{tabular}

DDWH = degenerative disc disease without herniation; Func $=$ functional; HID = herniated intervertebral disc; LS =lumbar strain; PLS = post laminectomy syndrome; NS = non-symp; Pat = pathological parameters. *'Others' includes facet arthropathy, lumbar fracture, lumbar spinal stenosis, lumbar contusion, osteoarthritis, spondylolysis, scoliosis, spondylolisthesis and other conditions 
protocol that was carried out in all patients in the sagittal, coronal and transverse planes.

\section{Test Sequence I:}

1. RoM with no resistance in 3-D.

2. Isometric test to define the maximal isometric torque (MIT) in 3-D.

3. Dynamic repetitive tests simultaneously measuring torque, speeds and RoM. The patient was instructed to move in one plane at a time (the primary) while simultaneously applying $25 \%$ or $50 \%$ of the MIT measured previously in that plane as resistance. Isometric torques (maximum torques in the two secondary planes, MST) were concomitantly measured in the two secondary planes. The test was repeated for all three planes.

\section{Test Sequence II:}

4. Repeated RoM measurement in 3-D with no resistance.

5. Repeated dynamic tests measuring speed and RoM as in test sequence 1 .

\section{Abnormal performance}

The parameters measured by the system were RoM, MIT, maximum velocity against $25 \%$ MIT and $50 \%$ MIT $\left(\mathrm{MV}_{25}\right.$ and $\mathrm{MV}_{50}$, respectively), and MST against $25 \%$ MIT and $50 \%$ MIT. The results (except for the RoM) were compared with those found in the normal volunteers database (Table 3). All torques (MIT and MST) and maximal speeds $\left(\mathrm{MV}_{25}, \mathrm{MV}_{50}\right)$ smaller than the 2.5th percentile of the normal value of the same sex were considered as abnormal.

Maximal isometric torques smaller than the 2.5th percentile of the normal value and maximal speeds smaller than the 5 th percentile of the normal value of the same sex were considered as abnormal indicators (AI) by the OOC. ${ }^{13}$ The maximal possible number of AIs in a test was 22: four in the category of maximal torques, six for maximal velocities and 12 for maximal secondary torques. The number of AI was calculated and reported.

The statistical methods used in this study were the single factor ANOVA and the student's $t$-test. All the measured and calculated parameters were analyzed. The four most important and independently measured parameters, the MIT, MV (both $\mathrm{MV}_{25}$ and $\mathrm{MV}_{50}$ ) and MST, are presented and discussed.

\section{Results}

We found our healthy sample to be compatible with the OOC normal data as reported elsewhere. ${ }^{13}$ The average number of AIs is presented in Table 1 together with the demographic data. The female patients had a significantly lower number of AIs than the males ( $t$ test, $P<0.001)$.

The OOC groups, both healthy controls and symptomatic, were of a similar age and height as our corresponding groups (Table 1). The only significant

Table 3 Main tested parameters in the OOC database, the non-symptomatic (NS) and symptomatic (S) patients

\begin{tabular}{|c|c|c|c|c|c|c|c|}
\hline Parameter & & $\begin{array}{c}O O C \\
F \\
(\text { Aver. } \pm \mathrm{SD})\end{array}$ & $\begin{array}{c}O O C \\
M \\
(\text { Aver. } \pm \mathrm{SD})\end{array}$ & $\begin{array}{c}N S \\
F \\
(\text { Aver. } \pm \mathrm{SD})\end{array}$ & $\begin{array}{c}N S \\
M \\
\text { (Aver. } \pm \mathrm{SD})\end{array}$ & $\begin{array}{c}S \\
F \\
(\text { Aver. } \pm \mathrm{SD})\end{array}$ & $\begin{array}{c}S \\
M \\
(\text { Aver. } \pm \mathrm{SD})\end{array}$ \\
\hline \multirow[t]{4}{*}{ MIT (Nm) } & Rot & $59 \pm 16$ & $101 \pm 22$ & $69 \pm 12$ & $113 \pm 34$ & $42 \pm 20$ & $60 \pm 34$ \\
\hline & $\mathrm{Fl}$ & $89 \pm 32$ & $177 \pm 48$ & $77 \pm 15$ & $172 \pm 55$ & $43 \pm 31$ & $70 \pm 51$ \\
\hline & Ex & $144 \pm 42$ & $230 \pm 54$ & $155 \pm 40$ & $222 \pm 71$ & $79 \pm 44$ & $96 \pm 62$ \\
\hline & $\mathrm{LF}$ & $102 \pm 27$ & $177 \pm 35$ & $97 \pm 25$ & $168 \pm 43$ & $61 \pm 31$ & $88 \pm 48$ \\
\hline \multirow[t]{3}{*}{$\mathrm{MV}_{25}(\mathrm{deg} / \mathrm{s})$} & Rot & $125 \pm 27$ & $152 \pm 32$ & $120 \pm 32$ & $175 \pm 51$ & $64 \pm 40$ & $64 \pm 40$ \\
\hline & $\mathrm{F} / \mathrm{E}$ & $143 \pm 28$ & $180 \pm 36$ & $141 \pm 16$ & $189 \pm 41$ & $75 \pm 47$ & $71 \pm 52$ \\
\hline & $\mathrm{LF}$ & $136 \pm 31$ & $158 \pm 42$ & $156 \pm 28$ & $173 \pm 48$ & $84 \pm 50$ & $77 \pm 56$ \\
\hline \multirow[t]{3}{*}{$\mathrm{MV}_{50}(\mathrm{deg} / \mathrm{s})$} & Rot & $98 \pm 26$ & $129 \pm 29$ & $86 \pm 28$ & $115 \pm 35$ & $46 \pm 28$ & $44 \pm 34$ \\
\hline & $\mathrm{F} / \mathrm{E}$ & $138 \pm 32$ & $161 \pm 37$ & $121 \pm 18$ & $156 \pm 34$ & $62 \pm 40$ & $58 \pm 44$ \\
\hline & $\mathrm{LF}$ & $108 \pm 24$ & $143 \pm 39$ & $111 \pm 17$ & $150 \pm 49$ & $61 \pm 36$ & $58 \pm 45$ \\
\hline \multirow[t]{3}{*}{ MSTrot* (Nm) } & Rot & - & - & - & - & - & - \\
\hline & $\mathrm{F} / \mathrm{E}$ & $46 \pm 19$ & $83 \pm 28$ & $42 \pm 15$ & $84 \pm 31$ & $26 \pm 15$ & $40 \pm 26$ \\
\hline & LF & $58 \pm 21$ & $77 \pm 15$ & $56 \pm 13$ & $83 \pm 18$ & $33 \pm 20$ & $42 \pm 25$ \\
\hline \multirow[t]{3}{*}{$\operatorname{MSTf} / \mathrm{e}^{*}(\mathrm{Nm})$} & Rot & $15 \pm 7$ & $21 \pm 10$ & $19 \pm 6$ & $28 \pm 12$ & $9 \pm 7$ & $11 \pm 9$ \\
\hline & $\mathrm{F} / \mathrm{E}$ & - & - & - & - & - & - \\
\hline & $\mathrm{LF}$ & $13 \pm 5$ & $19 \pm 9$ & $16 \pm 6$ & $22 \pm 9$ & $10 \pm 5$ & $13 \pm 9$ \\
\hline \multirow[t]{3}{*}{ MSTlf* (Nm) } & Rot & $39 \pm 12$ & $54 \pm 15$ & $41 \pm 14$ & $57 \pm 15$ & $22 \pm 16$ & $25 \pm 18$ \\
\hline & $\mathrm{F} / \mathrm{E}$ & $53 \pm 22$ & $94 \pm 30$ & $41 \pm 17$ & $88 \pm 36$ & $24 \pm 15$ & $38 \pm 25$ \\
\hline & $\mathrm{LF}$ & - & - & - & - & - & - \\
\hline
\end{tabular}

Rot $=$ rotation; $\mathrm{F} / \mathrm{E}=$ flexion/extension; $\mathrm{LF}=$ lateral flexion. See text for other abbreviations. *MST is presented while moving in the primary plane against $50 \%$ of maximal isometric torque 
difference was the higher weight of the OOC pathologic sample.

The tested groups were made of patients and volunteers with a variety of diagnoses (Table 2). Herniated intervertebral disc (HID) was the major diagnosis, with functional (Func) next.

The results cited and presented in Table 3 and Figures $1-4$ are the best results (highest MIT, $\mathrm{MV}_{25}$, $\mathrm{MV}_{50}$ and MST) from both test sequences. The differences between the results from the two sequences were non-significant and similar to those reported in the literature. ${ }^{13,14,20}$

Figures 1-4 present the results for the MIT, maximum speed $\left(\mathrm{MV}_{25}\right.$ and $\left.\mathrm{MV}_{50}\right)$ and maximal secondary torque (MST) in rotation (Rot), flexionextension $(\mathrm{F} / \mathrm{E})$, and lateral flexion (LF) in the LBP group and our non-symptomatic (NS) control group. The OOC test results were not significantly different from our non-symptomatic (NS) results (Table 3) and both were found to be significantly larger than the LBP group in all measured parameters.

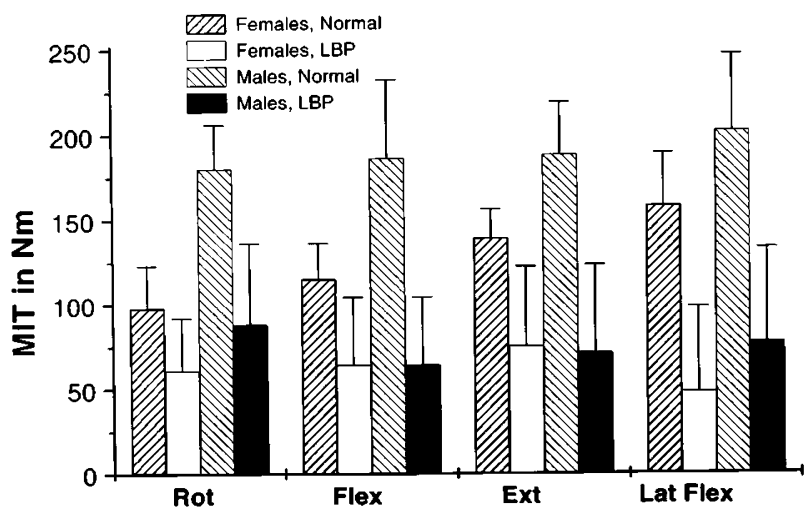

Figure 1 Maximal isometric torque (MIT) expressed in Newton*meter (Nm) in rotation (Rot), flexion (Flex), extension (Ext) and lateral flexion (Lat. Flex) in the study groups. The bars are representing standard deviations in all figures

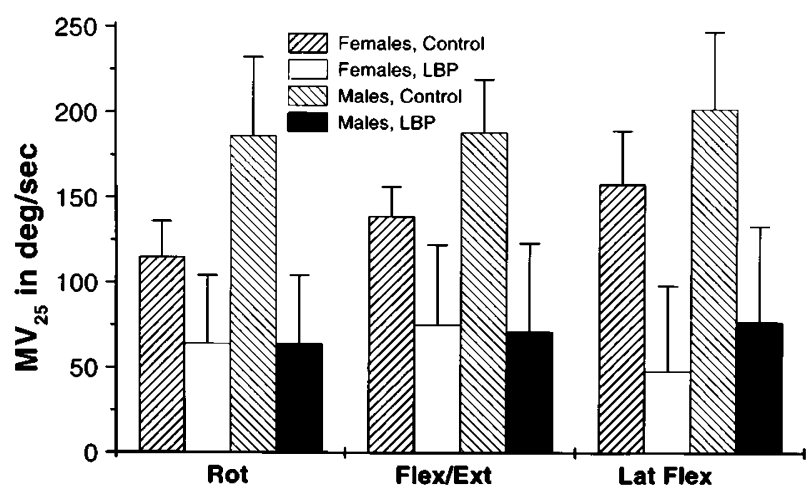

Figure 2 Maximal speed against $25 \%$ of maximal isometric torque $\left(\mathrm{MV}_{25}\right)$ in rotation (Rot), flexion/extension (Flex/Ext) and lateral flexion (Lat. Flex) for the study groups
Figure 1 shows the MIT in four directions for our LBP and NS groups. While the change between the NS and LBP groups is highly significant ( $t$-test, $P<0.001)$ in all planes, the change is larger among the males (Table 3) and especially so in flexion/ extension.

Figure 2 shows the $\mathrm{MV}_{25}$ in all directions. Again, the change between the NS and LBP groups is highly significant $(t$-test, $P<0.001)$ in all planes. The difference is larger between NS and LBP males, except for lateral flexion (Table 3). Very similar results were found for the $\mathrm{MV}_{50}$ in all directions (Figure 3 and Table 3).

The MST are demonstrated in Figure 4 and Table 3. The MST in the LBP males was significantly smaller than in the NS males $(P<0.001)$; the decrease was significantly smaller among the females $(P<0.01)$. The MST in rotation was the highest and in lateral flexion second. MST in flexion/extension was significantly $(P<0.001$, ANOVA) smaller.

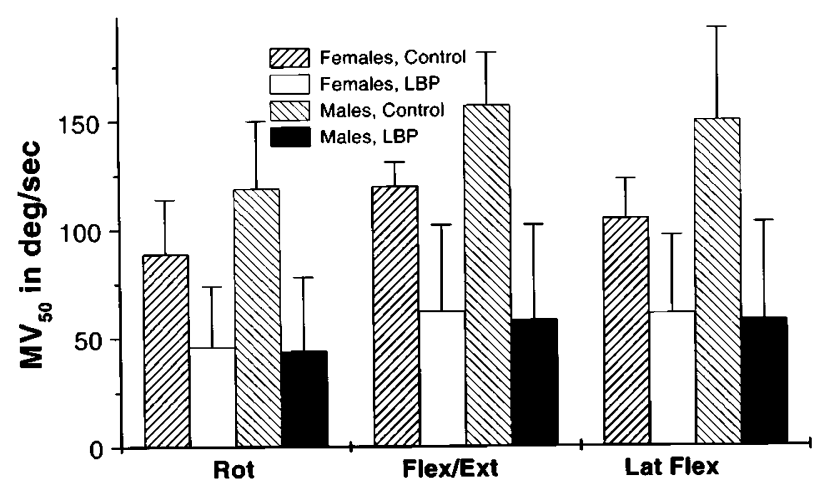

Figure 3 Maximal speed against $50 \%$ of maximal isometric torque $\left(\mathrm{MV}_{50}\right)$ in rotation (Rot), flexion/extension (Flex/Ext) and lateral flexion (Lat Flex) for the study groups

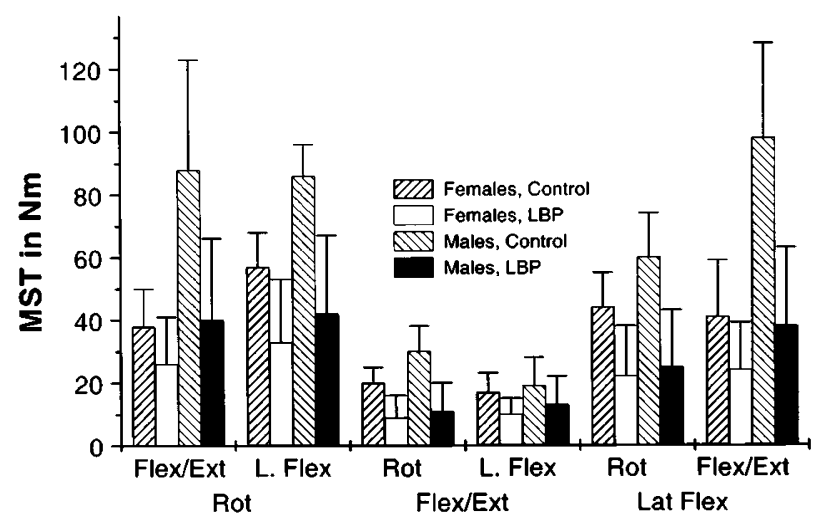

Figure 4 Maximal secondary torque (MST) in three planes. MST in the secondary direction (middle notation) is presented while moving in the prime direction (lower notation) against $50 \%$ maximal isometric torque 
In the present study, the patient group consisted of individuals who were referred to our institute and were defined as chronic LBP patients by their own physician. We found 558 of them to have at least one pathological parameter (average $12 \pm 7$ Pat), while 49 patients had none. Considering the clinician's assessments as accurate, this might indicate a sensitivity of more than $91 \%$. Thirty-four out of the 38 clinically healthy or NS subjects were identified correctly (ie, with no pathologic parameters), indicating a specificity of better than $89 \%$ considering the clinical findings accurate. The lack of 'golden standards' is a major methodological obstacle if one tries to assess the validity, sensitivity or specificity of any assessment method. We encountered the same problem while using the 3-D dynamometer method. All our patients were examined by at least two independent experts, but the truthfulness of the associated functional disability as presented by the patients was questioned due to possible expected secondary gains. The healthy, non-symptomatic volunteers were assessed by an expert clinician and their clinical findings were found to be within normal ranges. Comparing the clinical assessment of the tested subjects and the B-200 findings, the use of the parameters suggested by the OOC was found to be sensitive, useful and reliable.

The speed of movement - both the average and maximal values - is currently considered as one of the most reliable parameters describing low back condition and function. ${ }^{20}$ Our current study supported that observation. The maximal speeds changed significantly between the LBP patients and the NS subjects in all planes, being significantly larger in the latter (ANOVA, $P<0.001$ ).

The ability to measure synergistic muscle torques employed in secondary planes is one of the advantages of the IsoStation B-200. The prime mover muscles are always accompanied by synergistic muscles and both have secondary axis components. ${ }^{24}$ The synergetic activity is mostly subconscious and dictated mainly by anatomy and geometry, hence it represents a true and reliable parameter.

The relatively smaller maximal secondary torque (MST), found in healthy and unhealthy subjects alike during flexion/extension (Figure 4) might be explained, at least in part, by the anatomy of the major back muscles and a better voluntary control of muscles recruitment for this movement, performed most often and under full visual control.

The 'learning effect' was investigated by Hutten and Hermens $^{25}$ and found not to exist. Hence, the use of a single test per patient is sufficient and representative of his/hers disability. Since we did not have the opportunity to re-examine the same patients in the big majority of cases, we had to rely upon this finding as well as inter-test and inter-tester repeatability, reproducibility and reliability described in the literature by Spengler et $a l,{ }^{11}$ Szpalski et al, ${ }^{23}$ Parnianpour et $a l,{ }^{12}$ and others. They reported the repeatability, 
reproducibility and reliability to be good, supporting the use of the IsoStation B-200, the test protocol and the abnormal indicators.

There are numerous methods and techniques used in the anatomical and clinical evaluation of the patient suffering from LBP, including imaging and electromyography. However, the functional disability associated with the clinical and anatomical findings is a highly questionable matter. 5 The 3-D dynamometer used in the present study adds some useful objective information that can be of significant help to the physicians in their functional evaluation.

Evaluation with the 3-D dynamometer is not fully unraveled yet. The system enables the graphic display and analysis of all possible relations between the various parameters measured, and the significance of many of those is still being evaluated. The current database is limited and there is a need to increase it significantly in order to account for specific populations (eg, sportsmen). Many other studies are still needed, and there is a wide range of improvements and 'fine tuning' that should be carried out. However, even in its present stage, we contend that the IsoStation B-200 is a useful tool that contributes objective measurements to the otherwise largely subjective clinical assessment of LBP.

\section{Conclusions}

Considering the enormous social and economical ramifications, assessment of low back pain should be based on as objective and useful data as possible. The IsoStation B-200 is a system that can help supply such information. The highly significant differences found between the LBP patients and healthy subjects in all measured parameters support the use of the B-200 as a supplementary objective tool for functional LBP assessment, and the measured parameters as reliable indicators of abnormality.

\section{Acknowledgements}

The authors wish to thank Mrs Esther Eshkol for her assistance in preparing the manuscript.

\section{References}

1 Frymoyer JW. Orthopaedic Knowledge Update 4: Home Study Syllabus, 1993. AAOS. pp 491-493.

2 Bell N. Oh, my aching back! Business and Health, April 1991, pp $360-365$.

3 Spengler DM. Newer assessment approaches for the patient with low back pain. In: Rothman RH, Simeone FA (eds). The Spine, 3rd edn., WB Saunders, Philadelphia, 1992. pp $1921-1928$.
4 American Medical Association (AMA). Guides to the evaluation of permanent impairment. 4th edition. American Medical Ass., 1995.

5 Jensen MC, Brant-Zawadski MN, Obuchowski N et al. Magnetic resonance imaging of the lumbar spine in people without back pain. $N$ Engl J Med 1994; 331: 69-73.

6 Wiesel SW, Tsourmas N, Feffer HL et al. A study of computer assisted tomography. I. The incidence of positive CAT scans in an asymptomatic group of patients. Spine 1984; 9: 549-551.

7 Hirsch G et al. Relationship between performance on lumbar dynamometry and Waddell score in a population with low-back pain. Spine 1991; 16: 1039-1043.

8 Leclaire Richard et al. Diagnosis accuracy of technologies used in LBP assessment. Spine 1996; 21: 1325 - 1331.

9 Levene JA et al. Trends in isometric trunk testing on the IsoStation B200. J Spinal Disord 1989; 2: 20-35.

10 Tan JC et al. Isometric maximal and submaximal trunk extension at different flexed positions in standing. Spine 1993; 18: $2480-$ 2490.

11 Spengler DM, Szpalski M. Newer assessment approaches for the patient with low back pain. Contemp Orthop 1990; 21: 371-378.

12 Parnianpour M, Li F, Nordin M, Frankel VH. Reproducibility of trunk isoinertial performances in the sagittal, coronal and transverse planes. Bull Hos Jt Dis Orthop Inst 1989; 49: 148 - 154

13 B-200 Back Evaluation System (manual). Version 3.0. Occupational Orthopaedic Center (OOC), RI, USA, 1991.

14 Gomez $\mathrm{T}$ et al. Normative database for trunk range of motion, strength velocity and endurance with the IsoStation B-200 lumbar dynamometer. Spine 1991; 16: 15-21.

15 Rytokoski U et al. Measurement of LB mobility, isometric strength and isoinertial performance with Isostation B-200 triaxial dynamometry: reproducibility of measurement and development of functional indices. J Spinal Disord 1994; 7: 54 61

16 Parnianpour M, Nordin M, Kahanovotz N, Frankel V. The triaxial coupling of torque generation of trunk muscles during isometric exertions and the effect of fatiguing isoinertial movements on the motor output and movement patterns. Spine 1988; 13: $982-992$.

17 Szpalski M, Hayez J. How many days of bed rest for low back pain? Objective assessment of trunk function. Eur Spine J 1992; 1: $29-31$.

18 Nissan $\mathrm{M}$ et al. The normal, healthy low back: some functional parameters. J Back \& Musculoskeletal Rehabilitation 1999; 12: $1-5$.

19 Hutten MMR, Hermens HJ. Relationship between isoinertial lumbar dynamometry parameters and demographic parameters in chronic low back pain patients. Eur Spine J 1998; 7: 454-460.

20 Parnianpour M, Nordin M, Sheikhzadeh A. The relationship of torque, velocity and power with constant resistive load during sagittal trunk movement. Spine 1990; 15: 639-643.

21 Hayes B, Solyom C, Wing P et al. Use of psychometric measures and nonorganic signs testing in detecting nomogenic disorders in LBP patients. Spine 1993; 18: $1254-1262$.

22 Waddell G, McCulloch JA, Kummel E et al. Nonorganic physical signs in LBP. Spine 1980; 5: 117-125.

23 Szpalski M et al. Reproducibility of trunk isoinertial dynamic performance in patients with low back pain. J Spinal Disord 1992; 5: $78-85$.

24 Basmajian JV, De Luca CJ. Muscles Alive. Their Function Revealed by EM. 5th edn. Williams \& Wilkins, Baltimore. 1985.

25 Hutten MMR, Hermens HJ. Reliability of lumbar dynamometry measurements in patients with chronic low back pain with testretest measurements on different days. Eur Spine J 1997; 6: $54-$ 62. 